\title{
Autopsies and Asymptomatic Patients During the COVID-19 Pandemic: Balancing Risk and Reward
}

\author{
Matteo Nioi ${ }^{1 \star t}$, Pietro Emanuele Napoli ${ }^{2 \dagger}$, Maurizio Fossarello ${ }^{2}$ and Ernesto d'Aloja ${ }^{1+}$ \\ ${ }^{1}$ Forensic Medicine Unit, Department of Clinical Sciences and Public Health, University of Cagliari, Cagliari, Italy, \\ ${ }^{2}$ Department of Surgical Science, Eye Clinic, University of Cagliari, Cagliari, Italy
}

Keywords: COVID-19 pandemic, COVID-19 autopsy, COVID-19 autopsy asymptomatic, autopsy safety, CoVID-19 tests, SARS-CoV-2 cadaveric persistence, COVID-19 HCWs

\section{OPEN ACCESS}

Edited by:

George Dimopoulos,

University General Hospital

Attikon, Greece

Reviewed by:

Saad Nseir,

Centre Hospitalier Regional et

Universitaire de Lille, France

*Correspondence:

Matteo Nioi

nioimatteo@gmail.com

tThese authors have contributed equally to this work

Specialty section:

This article was submitted to Infectious Diseases - Surveillance,

Prevention and Treatment, a section of the journal

Frontiers in Public Health

Received: 21 August 2020

Accepted: 13 October 2020

Published: 09 November 2020

Citation:

Nioi M, Napoli PE, Fossarello $M$ and

d'Aloja E (2020) Autopsies and

Asymptomatic Patients During the

COVID-19 Pandemic: Balancing Risk and Reward.

Front. Public Health 8:595405.

doi: 10.3389/fpubh.2020.595405
Although we have gained much knowledge of coronavirus disease 2019 (COVID-19), its pathogenesis needs further investigation. Autopsies seem fundamental to confirming the Severe Acute Respiratory Syndrome-Coronavirus 2 (SARS-CoV-2) transmission pathways, pathogenetic mechanisms, and natural history.

The air and contact transmission routes of SARS-CoV-2 have been well-established, but further confirmatory work is needed on the oral-fecal, hematic, and ocular routes (1). Also, some aspects of the natural history of SARS-CoV-2 remain unclear. For example, what are the interindividual characteristics that determine the severity of COVID-19 among those infected with the virus?

The mechanisms by which SARS-CoV-2 infection leads to extrapulmonary manifestations are also unclear. Based on the symptoms affecting the gustatory and olfactory system, some authors have hypothesized an underlying neurogenic mechanism.

The pathogenesis of the important symptoms affecting the vascular system should also be defined. Some studies have suggested that it is these effects on the vascular system that are responsible for the thrombo-embolic events leading to the terminal phase of COVID-19 (2).

However, one of the most important unsolved questions concerns the very high number of asymptomatic patients. This category includes individuals who are positive for SARS-CoV-2 but with no clinical or radiological manifestations of COVID-19. Their percentage is estimated, in some preliminary studies, to be approximately $17.9 \%$ in a "closed" system. However, the real number of asymptomatic subjects is likely largely underestimated given the pandemic nature of COVID-19 and the low number of tests performed. Health policies have provided for a low number of autopsies and swabbing in subjects for whom there was no suspicion of COVID19. This prevents an understanding of whether positivity in SARS-CoV-2 can lead to subclinical pathological changes. Clarifying whether there are pathological or genetic factors (3) that can explain why some individuals are not susceptible to the virus or, when testing positive, do not develop the pathology is of fundamental importance.

Greater clarity on these issues would surely help when developing treatment and prophylaxis strategies for COVID-19.

These strategies could undoubtedly have therapeutic importance and could help guide weighted choices about how best to protect public health; asymptomatic patients are a potential source of contagion (4).

The existence of asymptomatic SARS-CoV-2 infection is an intriguing puzzle; it does represent a potential danger for those who perform autopsies.

Although transmission by asymptomatic patients is a crucial point in the ongoing pandemic, the guidelines for performing autopsies continue to underestimate the biological risk posed by 
such patients. The importance of the topic is relevant. In Italy, for example, several law firms are proposing lawsuits against health workers who treated the victims of COVID-19: this phenomenon will inevitably translate into an increase in autopsies on infected corpses (5).

The current indications concern deceased individuals "who have died of suspected or confirmed COVID-19" or in which the presence of the disease is considered "possible" (6).

Although several authors have proposed alternative solutions that might lower the risk of infection, these solutions do not guarantee that all cases will be intercepted. In the current context, the discoveries on the SARS-CoV-2 virus are extremely fast, and there is a risk that "provisional" indications conceived in the first phase may be outdated or require the introduction of an implementation in a few weeks or months. The indications given for the first phase were those used before the pandemic for diseases with a high risk of infection.

Thus, the recommendations included the use of autopsy rooms with a biological safety level of at least 3 (BSL-3). Another recommendation is an adequate use of personal protective equipment (PPE) including a surgical suit, headgear, visor, FFP3 mask, and waterproof gloves that extend up to the forearm with targeted use of oropharyngeal swabs. Another suggestion concerned particular sectoral techniques to be used in suspected cases such as manual opening of the skull and block sampling of heart and lungs. Among the most complete recommendations produced so far are those of Aquila et al. who hypothesize the possibility of carrying out a swab even on individuals in whom

\section{REFERENCES}

1. Napoli PE, Nioi M, d'Aloja E, Fossarello M. (2020). The ocular surface and the Coronavirus disease 2019: does a dual 'ocular route'exist? J Clin Med. 9:1269. doi: 10.3390/jcm9051269

2. Rothan HA, Byrareddy SN. (2020). The epidemiology and pathogenesis of coronavirus disease (COVID-19) outbreak. J Autoimmun. 109:102433. doi: 10.1016/j.jaut.2020.102433

3. Napoli PE, Nioi M. (2020). Global spread of coronavirus disease 2019 and malaria: an epidemiological paradox in the early stage of a pandemic. J Clin Med. 9:1138. doi: 10.3390/jcm9041138

4. Bai Y, Yao L, Wei T, Tian F, Jin DY, Chen L, et al. (2020). Presumed asymptomatic carrier transmission of COVID-19. JAMA. 323:1406-7. doi: 10.1001/jama.2020.2565

5. d'Aloja E, Finco G, Demontis R, Napoli PE, Fossarello M, Nioi M. (2020). COVID-19 and medical liability: Italy denies the shield to its heroes. EClinicalMedicine. 25:100470. doi: 10.1016/j.eclinm.2020.10 0470

6. WHO. Infection Prevention and Control for the Safe Management of a Dead Body in the Context of COVID-19. (2020). Available online at: https://apps.who.int/iris/bitstream/handle/10665/331538/WHO-COVID19-IPC_DBMgmt-2020.1-eng.pdf

7. Aquila I, Sacco MA, Abenavoli L, Malara N, Arena V, Grassi S, et al. SARS-CoV-2 pandemic: review of the literature and proposal there is no suspicion of COVID-19 with consequent adjustments to the procedure in the event of a positivity. However, even this guide does not consider the pre-autoptic phase (judicial inspection), the post-autoptic phase (transport from the sectoral room to the burial place), and the analytical limits linked to the swab $(7,8)$.

The sensitivity of reverse transcriptase-PCR (RT-PCR) tests of throat and pharyngeal swabs has been estimated at around $70 \%$. (9) Even the most sensitive serological tests are unable to intercept positivity in the early stages of infection (10).

Finally, it should be noted that these tests have not been validated on postmortem samples, and the persistence time of the virus in affected corpses remains-to our knowledge-undetermined.

To conclude, we believe that the widespread and systematic execution of autopsies will contribute to our knowledge of COVID-19. However, caution is needed, and for now, all corpses should be treated as a source of SARS-CoV-2, regardless of swab or serological test results.

\section{AUTHOR CONTRIBUTIONS}

MN, Ed'A, and PN conceived of the presented idea. MF developed the theory. $\mathrm{MN}$ wrote the manuscript in consultation with Ed'A, PN, and MF. All authors contributed to the article and approved the submitted version. for safe autopsy practice. Arch Pathol Lab Med. (2020) 144:104856. doi: 10.5858/arpa.2020-0165-SA

8. Napoli PE, Nioi M, d'Aloja E, Fossarello M. Safety recommendations and medical liability in ocular surgery during the COVID-19 pandemic: an unsolved dilemma. J Clin Med. (2020) 9:1403. doi: 10.3390/jcm9051403

9. Pondaven-Letourmy S, Alvin F, Boumghit Y, Simon F. How to perform a nasopharyngeal swab in adults and children in the COVID-19 era. Eur Ann Otorhinolaryngol Head Neck Dis. (2020) 137:325-7. doi: 10.1016/j.anorl.2020.06.001

10. Lai CC, Wang CY, Ko WC, Hsueh PR. In vitro diagnostics of coronavirus disease 2019: technologies and application. J Microbiol Immunol Infect. (2020). doi: 10.1016/j.jmii.2020.05.016. [Epub ahead of print].

Conflict of Interest: The authors declare that the research was conducted in the absence of any commercial or financial relationships that could be construed as a potential conflict of interest.

Copyright (C) 2020 Nioi, Napoli, Fossarello and d'Aloja. This is an open-access article distributed under the terms of the Creative Commons Attribution License (CC BY). The use, distribution or reproduction in other forums is permitted, provided the original author(s) and the copyright owner(s) are credited and that the original publication in this journal is cited, in accordance with accepted academic practice. No use, distribution or reproduction is permitted which does not comply with these terms. 